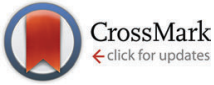

Cite this: J. Mater. Chem. C, 2016 4, 2936

Received 13th October 2015,

Accepted 12th December 2015

DOI: $10.1039 / \mathrm{c5tc03311j}$

www.rsc.org/MaterialsC

\section{Tuning the aggregation-induced enhanced emission behavior and self-assembly of phosphole-lipids $\dagger$}

\author{
Zisu Wang, Benjamin S. Gelfand, Pengcheng Dong, Simon Trudel and \\ Thomas Baumgartner*
}

\begin{abstract}
Herein, we report on the synthesis, self-assembly, as well as the photophysical properties of a novel series of $P$-benzylated phosphole-lipids. In the context of this structure-property study we have systematically altered the number, position, and length of the alkyl chains in the 3-,4-, and 5-position of the benzyl group. Both the self-assembly and photophysical properties of the compounds were found to correlate strongly with the alkyl chain length and chain arrangement of the mesogenic moiety.
\end{abstract}

\section{Introduction}

Organic materials that contain main group elements, such as $\mathrm{P},{ }^{1 a-d} \mathrm{~B},{ }^{1 e, f}$ and $\mathrm{Si}^{1 g}$ are experiencing a surge in interest. This is due to the unique structural and electronic properties these elements can impart on the organic scaffold of the material.

Among structural motifs commonly found in organo-main group molecules, phospholes are a unique class of compounds. ${ }^{1 a}$ Distinct from the analogous pyrroles, phospholes are partially aromatic, as the phosphorus lone pair has only little overlap with the $\pi$ orbital of the backbone. ${ }^{1 a}$ The limited aromaticity of the molecule actually comes from the interaction of the $\sigma^{*}$ orbital of the P-R single bond and the $\pi^{*}$ orbital of the conjugated scaffold (Fig. 1). Due to these unique interactions, chemical modification of the phosphole lone pair can have a considerable effect on the optoelectronic properties of the resulting compounds. By adjusting the substituent ' $\mathrm{E}$ ' on the lone pair, the emission properties of the compounds can be easily tuned without the need for modification of the backbone. ${ }^{2}$

Over more than a decade, our group has been involved with the development of functional conjugated organophosphorus species and we have established various highly desirable optoelectronic features for the materials. ${ }^{3}$ To expand the general utility of such species in a materials context, we have recently reported on a series of self-assembled dithienophospholebased materials. These 'phosphole-lipids' exhibit liquid crystalline properties over a wide temperature range (Fig. 2). ${ }^{4 a}$

Department of Chemistry and Centre for Advanced Solar Materials, University of Calgary, 2500 University Drive NW, Calgary, Alberta T2N 1N4, Canada.

E-mail: thomas.baumgartner@ucalgary.ca

$\dagger$ Electronic supplementary information (ESI) available. CCDC 1431111. For ESI and crystallographic data in CIF or other electronic format see DOI: 10.1039/ c5tc03311j a

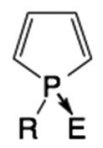

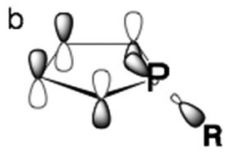

Fig. 1 The unique electronics of phospholes. (a) General bonding patterns of phospholes; (b) $\sigma^{\star}-\pi^{\star}$ interaction in phospholes.

Some of the phosphole-lipids also display gelation properties in a wide range of solvents at low concentrations. ${ }^{2 b}$ The selfassembly features of phosphole-lipids are the results of a balancing act between intermolecular $\pi-\pi$ interactions of the phosphole backbones, the intermolecular ionic interactions and the thermal disorder imparted by the flexible alkyl chains. As can be seen in Fig. 2, with larger $\pi$ conjugation in its phosphole backbone, the increased intermolecular $\pi-\pi$ interactions in 1a led to a more crystalline soft crystal phase unlike the liquid crystal phase found in the smaller DTP. Therefore, we became interested in further exploring this delicate balance between the driving forces and tuning the self-assembly feature of our phosphole-lipid system in more detail.

In phosphole-lipids, quaternization at the phosphorus lone pair affords the ionic feature of the compounds, which is a critical directing force for the self-assembly process. At the same time, due to the aforementioned $\sigma^{*}-\pi^{*}$ hyperconjugation, the benzyl moiety also heavily participates in the optical transitions of the molecule. As such, the rotational flexibility of the benzyl moiety also impacts the optoelectronic behavior of the compounds both in solution and in the solid state. ${ }^{4 a, c} \mathrm{We}$ were further able to confirm that stimuli-responsive features of phosphole-lipids such as aggregation-induced enhanced emission (AIEE) and mechanochromism also stem from this particular role of the benzyl moiety. ${ }^{4}$ 

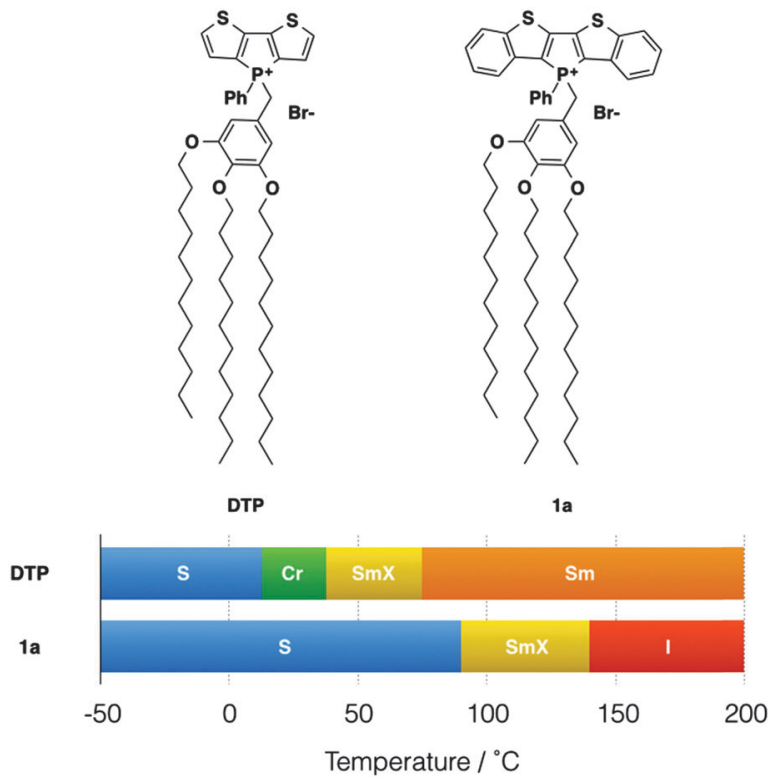

Fig. 2 Phosphole-lipid liquid crystals. S, "solid"; Cr, crystalline state; SmX, soft crystal; Sm, smectic phase; I, isotropic liquid.

AIEE is the phenomenon where the aggregated-state fluorescence quantum yield of the compound exceeds that of the compound in solution. ${ }^{5}$ Contrary to the more common aggregation-caused quenching (ACQ) of fluorescence that is found in organic chromophores, AIEE-enabled materials hold more appeal in application settings such as OLED displays. As well, due to the increased fluorescence of the compounds in the solid state, these materials provide interesting opportunities in studying the photoluminescence process in the solid state. ${ }^{5}$ Tetraphenylethylenecontaining compounds and phenyl-appended siloles are two of the most studied AIEE systems for their excellent response to concentration variations not only in solution, but also in the solid state (Fig. 3). ${ }^{5}$ The AIEE in both systems stems from the free/restricted intramolecular motion of conjugated peripheral substituents.

While the general principle of AIEE can be understood with relative ease, the effect of self-organization on AIEE still needs to be better defined. To this end, the well defined self-assembly features of the phosphole-lipid system enable us to gain a glimpse into the relationship between self-assembly and AIEE.

Herein, we report on our efforts in further exploring and enhancing both the self-assembly and the AIEE properties of phosphole-lipids. In the context of this study, the di(benzothieno)phosphole-based lipid was chosen because of the promising packing features of the $\pi$-extended head group. ${ }^{4}$ a

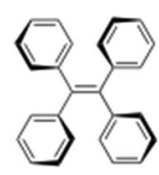

b

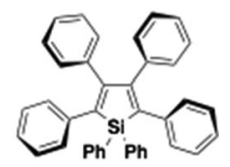

Fig. 3 Examples of common AIEE chromophores. (a) Tetraphenylethylene; (b) hexaphenylsilole.

\section{Results and discussion}

\section{Synthesis}

To gain deeper insights into the underlying parameters required for the self-assembly and ultimately the AIEE of the phosphole-lipid system, a variety of target compounds were chosen for this study, in which we have selectively altered the number and position of the benzyl alkyl chains as well as their length (Scheme 1).

The dodecyl-functionalized species (2a, 3a and 4a) were aimed at paralleling and expanding our original studies ${ }^{4}$ in order to see if a different placement of the dodecyl chains led to distinct self-assembly behavior and/or altered electronics. To this end, we expected to pinpoint and systematically study the effect of chain arrangements on the resulting solid-state properties. The octyl-functionalized species (1), 2b, 3b and $\mathbf{4 b}$ ) were targeted to study the effects of a shifted balance with regard the potential intra- and intermolecular interactions via van-der-Waals and $\pi$-stacking interactions.

The di(benzothieno)phosphole $\mathbf{S 1}$ was synthesized following our reported procedure. ${ }^{6}$ The various alkoxybenzyl bromides S2 were obtained in high yields by following the standard procedure reported by Zhao et $a l^{7}$ Quaternization of the phosphorus center afforded the target compounds in modest to good yields (Scheme 1).

To also test the counteranion as effective handle for tuning the self-assembly properties of phosphole-lipids, a model study was conducted with the cation of $\mathbf{4 a}$ and the dodecylsulfate anion. Dodecylsulfate was chosen because its ability to promote amphiphilic properties ${ }^{8}$ and its expected compatibility with the dodecyl chains already present in the cationic portion of $\mathbf{4 a}$. The exchange of the bromide for dodecylsulfate was accomplished by repeated aqueous extraction of an equimolar solution of the phosphole-lipid $4 \mathbf{a}$ and sodium dodecylsulfate $\left(\mathrm{Na}^{+} \mathrm{DS}^{-}\right)$ in dichloromethane to yield the anion-exchanged species 4a-DS in $81 \%$ yield.

\section{Photophysical properties}

UV-Vis absorption spectra. To establish a baseline for the photophysics of the new compounds, UV-Vis absorption spectra in dichloromethane solution were collected on all

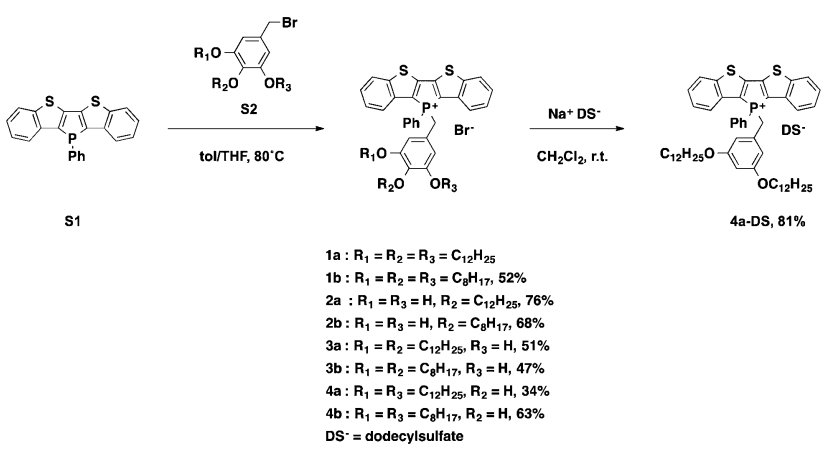

Scheme 1 Synthesis of phosphole-lipids. Quaternization of $\mathbf{S} 1$ with various benzyl bromides (S2) afforded the desired phosphole-lipids $\mathbf{1 - 4}$. The bromide anion was exchanged with dodecylsulfate via metathesis. 
Table 1 Photophysical features of the phosphole-lipids

\begin{tabular}{llllllrr}
\hline Compound & $\lambda_{\text {abs }}(\mathrm{nm})$ & $\log \varepsilon^{a}$ & $\lambda_{\text {em solution }}(\mathrm{nm})$ & $\phi_{\text {PL solution }}{ }^{b}$ & $\lambda_{\text {em solid-state }}(\mathrm{nm})$ & $\phi_{\text {PL solid-state }}{ }^{c}$ & AIEE response $^{d}(\%)$ \\
\hline 1b & 350,415 & $4.02,3.88$ & $\mathrm{n} / \mathrm{a}^{e}$ & 0.00 & $\mathrm{n} / \mathrm{a}^{e}$ & 0.00 & 0 \\
2a & 350,416 & $4.12,3.99$ & 514 & 0.26 & 526 & 0.03 & -23 \\
2b & 350,415 & $4.11,3.96$ & 512 & 0.26 & 529 & 0.05 & -21 \\
3a & 347,413 & $3.98,3.86$ & $\mathrm{n} / \mathrm{a}^{e}$ & 0.00 & $\mathrm{n} / \mathrm{a}^{e}$ & 0.00 & 0.09 \\
3b & 348,414 & $4.07,3.96$ & $\mathrm{n} / \mathrm{a}^{e}$ & 0.00 & 529 & 0.33 & 9 \\
4a & 347,417 & $4.05,3.90$ & 524 & 0.13 & 517 & 0.19 & -19 \\
4b & 349,416 & $4.07,3.92$ & 522 & 0.10 & 518 & 0.10 & -7 \\
4a-DS & 348,417 & $4.04,3.90$ & 524 & 0.17 & 529 &
\end{tabular}

${ }^{a}$ Molar absorption coefficient $\left(\mathrm{M}^{-1} \mathrm{~cm}^{-1}\right) .{ }^{b}$ Relative, measured against a quinine sulfate standard adjusted to the same absorbance, $\lambda_{\mathrm{ex}}=365 \mathrm{~nm}$. ${ }^{c}$ Absolute, determined using an integrating sphere, $\lambda_{\mathrm{ex}}=365 \mathrm{~nm} .{ }^{d}$ Obtained by subtracting $\phi_{\mathrm{PL} \text { solution }}$ from $\phi_{\mathrm{PL} \text { solid-state. Negative value indicates }}$ aggregation quenching, positive value indicates AIEE. ${ }^{e}$ Not available due to low signal to noise ratio.

compounds (Table 1). As can be seen in Fig. 4a, the absorption spectra of all the phosphole-lipid species are quite similar; two absorption peaks are observed (at approx. $350 \mathrm{~nm}$ and $415 \mathrm{~nm}$, respectively). The extinction coefficients of the compounds range between 0.9 and $1.3 \times 10^{4} \mathrm{M}^{-1} \mathrm{~cm}^{-1}(350 \mathrm{~nm})$, or 0.75 and $1 \times 10^{4} \mathrm{M}^{-1} \mathrm{~cm}^{-1}$ (415 nm), respectively (Fig. 4a).

Solution fluorescence spectra. Solution fluorescence spectra were also collected for all compounds, as it is a key component of AIEE (Table 1, Fig. 4b). Compared to the previously studied 1a, ${ }^{4 a}$ a few of the newly synthesized phosphole-lipids are significantly more fluorescent in solution. The 2-series of compounds, with only one chain in the 4-benzyl position, is among the most
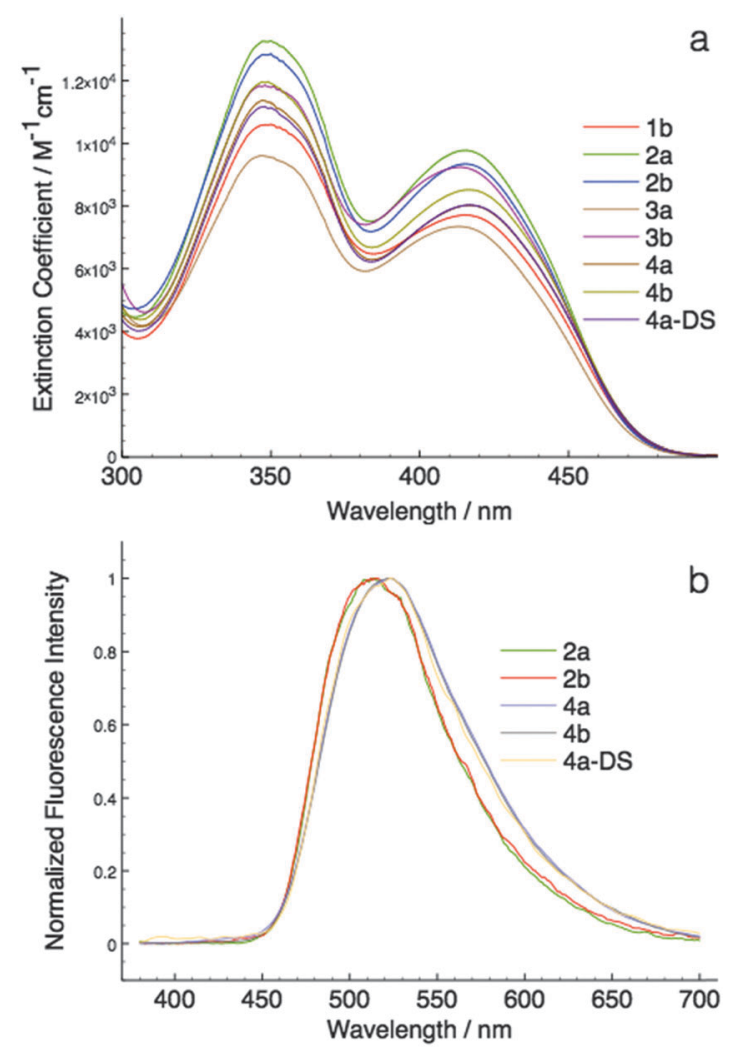

Fig. 4 Solution photophysics of phosphole-lipids. (a) UV-Vis absorption spectra of the phosphole-lipids; (b) fluorescence spectra of phospholelipids (low emission species was omitted for clarity due to low signal to noise ratio). highly fluorescent phosphole-lipids in solution studied to date (2a $\phi_{\mathrm{PL}}=26 \%, 2 \mathbf{b} \phi_{\mathrm{PL}}=26 \%$ ). The 4-series of compounds, with two chains in the 3,5-positions, is also quite fluorescent (4a $\phi_{\mathrm{PL}}=13 \%, \mathbf{4 b} \phi_{\mathrm{PL}}=9 \%$, 4a-DS $\left.\phi_{\mathrm{PL}}=16 \%\right)$. However, despite the highly analogous structural elements to the 4-series, the 3-series of compounds, with 3,4-positioned chains, is essentially non-emissive in solution. This contrast between the 3 - and 4-series was puzzling at first given their similar structure. Compound 1b mirrors the original 1a species in its low emissive properties in solution. $^{4 a}$

\section{Theoretical calculations}

Mechanism of AIEE in phosphole-lipids. The AIEE features of the phosphole-lipids relate intimately to the frontier molecular orbitals (FMOs) of the molecules. ${ }^{4}$ In an earlier report, ${ }^{4 a}$ the calculated FMOs of MS and M1 (Fig. 5, in which the alkyl chains are truncated by simple methyl groups) were used to illustrate the mechanism of AIEE in the phosphole-lipid system. The spatial separation of the Highest Occupied Molecular Orbital (HOMO; primarily the $\pi$ orbital of the benzyl moiety) and the Lowest Unoccupied Molecular Orbital (LUMO; primarily the $\pi^{*}$ orbital of the dithienophosphole head group) is the key to the AIEE of MS and related compounds. In MS, after a

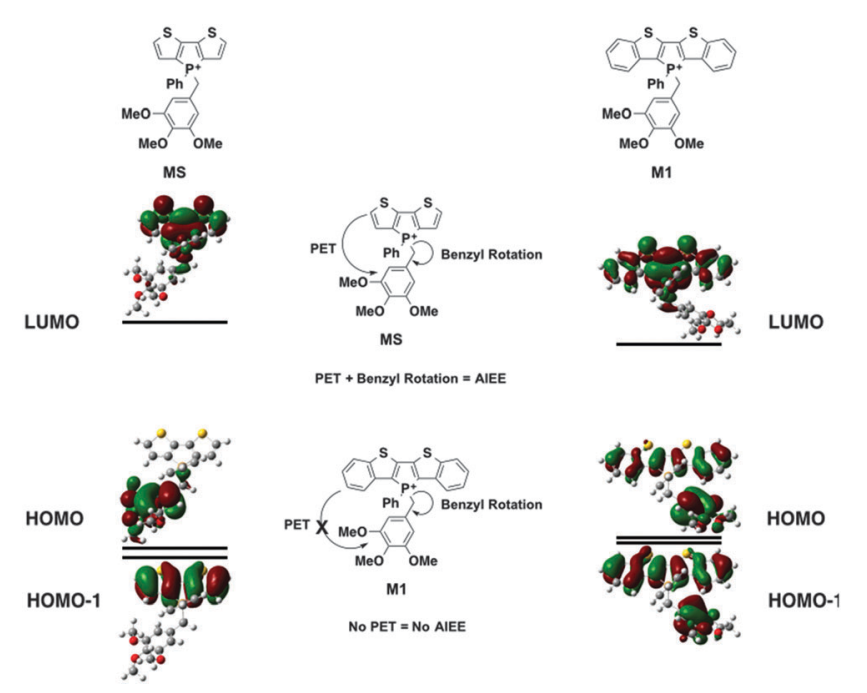

Fig. 5 Mechanism of AIEE in phosphole-lipids. 
photo-excitation from the HOMO into the LUMO, the relaxation process also involves the transfer of electron density spatially through the methylene linker at the benzylic position (photoinduced electron transfer, PET). The coupling between the relaxation process and the rotation of the benzylic methylene opens up non-radiative decay pathways and leads to quenching of fluorescence in solution. In the solid state, however, the restriction of the benzylic rotation leads to enhanced fluorescence in the case of MS. This is the basis of AIEE in the phosphole-lipid system. ${ }^{4 a}$

In M1, however, significant overlap exists between the FMOs of the species (Fig. 5). The PET process is therefore suppressed in this case, which leads to the lack of AIEE observed in the previously reported 1a species.

Static DFT calculations. To gain insights into the frontier orbital energies and the optical transitions of the di(benzothieno)phosphole-lipids in this study and to provide a rationalization for the peculiar emission properties of the compounds in solution, theoretical calculations at the $\mathrm{B} 3 \mathrm{LYP} / 6-31 \mathrm{G}+(\mathrm{d})$ level of theory $(\mathrm{PCM}$ solvation model in $\mathrm{CH}_{2} \mathrm{Cl}_{2}$ ) were conducted on model molecules M2, M3 and M4 using the Gaussian09 suite of programs (Fig. 6). ${ }^{9}$

Contrary to the observed photophysics, M3 and M4 are electronically quite similar to each other according to the calculations. The LUMO (primarily the $\pi^{*}$ orbital of the conjugated phosphole head group), HOMO (primarily the $\pi$ orbital of the benzyl moiety) and HOMO-1 (primarily the $\pi$ orbital of the phosphole head group) all have the same distribution in both species. A minor stabilization of the HOMO exists in M4, which can be attributed to the weaker $\pi$-donating nature of the meta-alkoxy substituent. Similar to MS, M3 and M4 are both PET-enabled making them strong candidates of AIEE features. Combining this result with the solution fluorescence observations, we can tentatively draw the conclusion that the significantly enhanced emissions of the 4 compounds do not originate from electronics but rather from different self-assembly in solution.

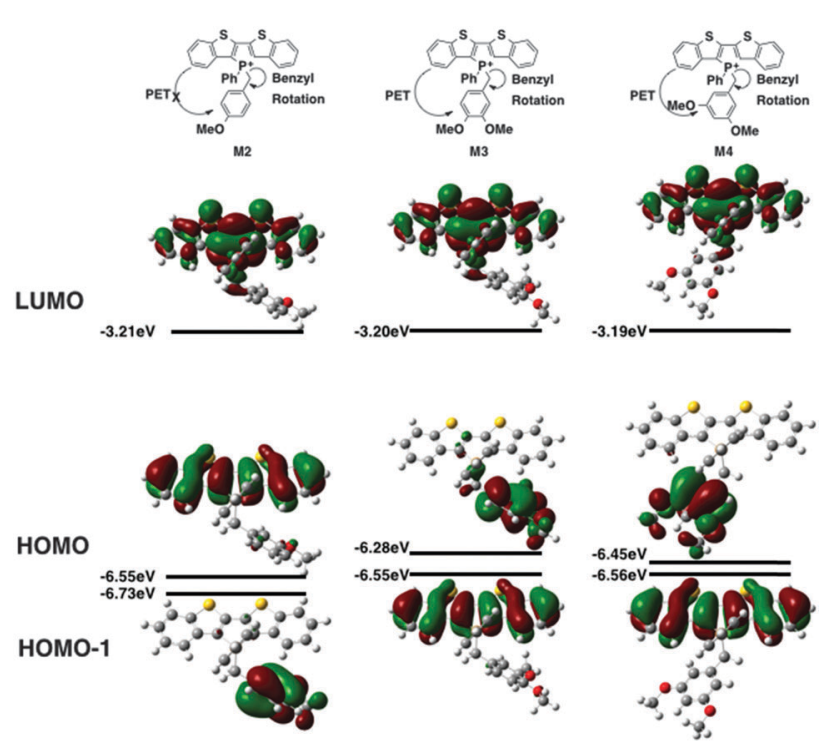

Fig. 6 Frontier orbitals of model phosphole-lipids.
M2, on the other hand, deviates significantly from the other species in this study (Fig. 6). Both its HOMO and LUMO reside mostly on the $\pi$ backbone of the conjugated phosphole head group. In addition, while spatial separation exists between HOMO-1 and LUMO in M2, the weak donating character of a single alkoxy substituent leads to a significant stabilization of HOMO-1, creating a considerable energy gap between HOMO and HOMO-1. As a result of the energy difference, the LUMO $\rightarrow$ HOMO-1 fluorescence transition is suppressed. Consequently, the compounds should behave much like other non-AIEE chromophores where the solution fluorescence is high. This prediction fits rather well with the observed high fluorescence of the compounds in solution.

Since the AIEE mechanism relies on the delicate energy balance between various orbitals, the conformation of the alkoxy substituents on the benzyl moiety was also investigated. The optimized structure (M4) placed the alkoxy group in plane with the benzene ring, which is quite reasonable as this conformation permits the conjugation between the $\mathrm{p}$ orbitals of the oxygen and the benzene ring. This conformation is also observed in the single crystal X-ray data of compound $4 \mathbf{a}$ (vide infra). DFT calculations were also conducted based on the X-ray crystallographic data of 4a (Fig. 7, M4-XRD with the dodecyl chains truncated to methyl for efficiency). With the endo-conformation of the benzyl substituent, there exists significant orbital communication between the benzyl substituent and the phosphole backbone. As a result, both the HOMO and the HOMO-1 were slightly stabilized compared to the optimized structure. This enlargement of the HOMO-LUMO gap was reflected in the slightly blue-shifted fluorescence of $\mathbf{4 a}$ in the solid state as compared to the solution $(\Delta \lambda=7 \mathrm{~nm})$. To our surprise, a profound effect was observed, when the alkoxy group was rotated $90^{\circ}$ with respect to the benzene plane, suppressing the lone pair $\pi$-conjugation (Fig. 7, M4-alkoxy rotated). The lack of $\pi$ donation

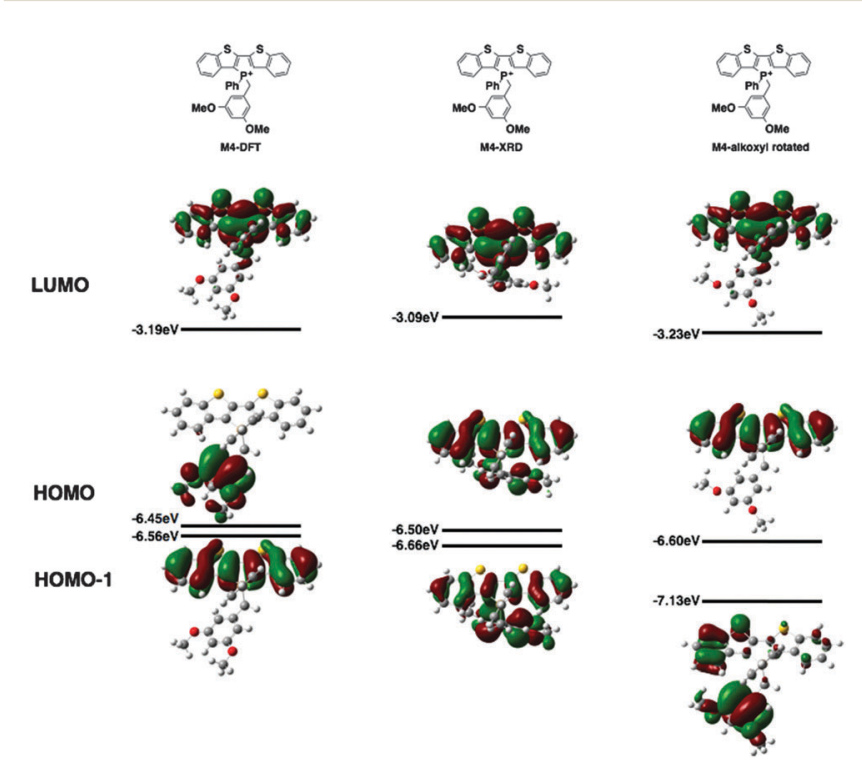

Fig. 7 DFT calculations of M4 as optimized (left), based on X-ray data (middle) and M4 with the alkoxy groups rotated (right). 
results in a significant stabilization $(-0.58 \mathrm{eV})$ of the benzyl $\pi$ orbital, which leads to the promotion of the phosphole $\pi$ orbital to become the HOMO. In this configuration, the AIEE should be suppressed. However, considering the possibility of somewhat free rotation of the alkoxy groups in solution (at least to some extent), would explain the observed AIEE, but also accounts in part the residual fluorescence of compounds $\mathbf{4 a}, \mathbf{b}$ in solution. This finding also indirectly supports the distinct emission features of the 3-series in solution, as the orthorelationship of the two alkoxy chains seems to restrict their free rotation, in contrast to the 3,5-arrangement in $\mathbf{4 a , b}$.

Time-dependent DFT calculations. While static calculations can provide a basis for discussion of the photophysical features of the compounds, time-dependent (TD) DFT calculations are needed to gain the full picture regarding the dynamic opto-electronic transition processes. The calculated oscillator strengths and the nature of the key transitions are summarized in Table 2.

As can be seen in Fig. 6, the HOMO-LUMO energy gaps appear to be quite varied. However, the UV-Vis spectra of the compounds were rather similar to each other (Fig. 4a). This discrepancy can be easily resolved by considering the TD-DFT calculation results. According to TD-DFT, both the low energy

Table 2 Calculated key photo-transitions

\begin{tabular}{|c|c|c|}
\hline & Calculated absorption bands & Nature of the transition \\
\hline M1 & $\begin{array}{l}\text { HOMO } \rightarrow \text { LUMO }(95 \%)^{a} \\
\text { HOMO-1 } \rightarrow \text { LUMO }(4 \%) \\
\left(436 \mathrm{~nm}, f^{p}=0.2821\right) \\
\text { HOMO-3 } \rightarrow \text { LUMO }(88 \%)^{a} \\
\text { HOMO-4 } \rightarrow \text { LUMO }(10 \%) \\
\left(358 \mathrm{~nm}, f^{b}=0.1636\right) \\
\text { HOMO-4 } \rightarrow \text { LUMO }(88 \%)^{a} \\
\text { HOMO-3 } \rightarrow \text { LUMO }(10 \%) \\
\left(352 \mathrm{~nm}, f^{b}=0.1226\right)\end{array}$ & $\begin{array}{l}\pi_{\text {phos }}-\pi_{\text {phos }}^{*} \\
\pi_{\text {benzyl }}-\pi_{\text {phos }}^{*} \\
\pi_{\text {phos }}-\pi^{*}{ }_{\text {phos }}\end{array}$ \\
\hline M2 & $\begin{array}{l}\text { HOMO } \rightarrow \text { LUMO }(99 \%)^{a} \\
\left(441 \mathrm{~nm}, f^{b}=0.2657\right) \\
\text { HOMO-2 } \rightarrow \text { LUMO }(97 \%) \\
(360 \mathrm{~nm}, f=0.1252) \\
\text { HOMO-3 } \rightarrow \text { LUMO }(97 \%) \\
(358 \mathrm{~nm}, f=0.182)\end{array}$ & $\begin{array}{l}\pi_{\text {phos }}-\pi_{\text {phos }}^{*} \\
\pi_{\text {phos }}-\pi_{\text {phos }}^{*} \\
\pi_{\text {benzyl }}-\pi_{\text {phos }}^{*} \\
\pi_{\text {phos }}-\pi_{\text {phos }}^{*}\end{array}$ \\
\hline M3 & $\begin{array}{l}\text { HOMO-1 } \rightarrow \text { LUMO }(99 \%) \\
(439 \mathrm{~nm}, f=0.2889) \\
\text { HOMO-2 } \rightarrow \text { LUMO }(98 \%) \\
(364 \mathrm{~nm}, f=0.077) \\
\text { HOMO-3 } \rightarrow \text { LUMO }(97 \%) \\
(357 \mathrm{~nm}, f=0.1814) \\
\text { HOMO-4 } \rightarrow \text { LUMO } \\
(347 \mathrm{~nm}, f=0.04)\end{array}$ & $\begin{array}{l}\pi_{\text {phos }}-\pi_{\text {phos }}^{*} \\
\pi_{\text {phos }}-\pi_{\text {phos }}^{*} \\
\pi_{\text {benzyl }} \pi^{*}{ }_{\text {phos }} \\
\pi_{\text {phos }}-\pi_{\text {phos }}^{*} \\
\pi_{\text {phos }}-\pi_{\text {phos }}^{*} \\
\pi_{\text {benzyl }}-\pi_{\text {phos }}^{*}\end{array}$ \\
\hline M4 & $\begin{array}{l}\text { HOMO-1 } \rightarrow \text { LUMO }(99 \%) \\
(435 \mathrm{~nm}, f=0.2818) \\
\text { HOMO-2 } \rightarrow \text { LUMO }(94 \%) \\
\text { HOMO-3 } \rightarrow \text { LUMO }(3 \%) \\
(363 \mathrm{~nm}, f=0.1355) \\
\text { HOMO-2 } \rightarrow \text { LUMO }(2 \%) \\
\text { HOMO-3 } \rightarrow \text { LUMO }(93 \%) \\
\text { HOMO-4 } \rightarrow \text { LUMO }(2 \%) \\
(357 \mathrm{~nm}, f=0.1849)\end{array}$ & $\begin{array}{l}\pi_{\text {phos }}-\pi_{\text {phos }}^{*} \\
\pi_{\text {phos }}-\pi_{\text {phos }}^{*} \\
\pi_{\text {benzyl }}-\pi_{\text {phos }}^{*} \\
\pi_{\text {phos }}-\pi_{\text {phos }}^{*} \\
\pi_{\text {benzyl }}-\pi_{\text {phos }}^{*}\end{array}$ \\
\hline
\end{tabular}

${ }^{a}$ Contribution of the particular transition to the absorption band. ${ }^{b} f=$ oscillator strength of the transition as calculated by TD-DFT. $(\sim 415 \mathrm{~nm})$ and the high-energy $(\sim 350 \mathrm{~nm})$ absorption bands are dominated by the $\pi-\pi^{*}$ transition of the phosphole head group. Benzyl involvements were rather limited in the excitation processes. Since the di(benzothieno)phosphole head groups are the same in all compounds, it is no surprise that the absorption spectra are similar in all cases.

\section{Solvatochromism}

To substantiate the PET process in the new phosphole-lipids, solvatochromism studies were carried out for two representative species $\mathbf{2 a}$ and $\mathbf{4 a}$. These compounds were chosen for their contrasting FMO arrangements (Fig. 6, M2 and M4).

Since the intramolecular PET process relies on the polar charge-separated excited state, polar solvents are more capable of stabilizing the excited state. Therefore it follows that, with a polar solvent, a bathochromic shift of the absorption peaks should be observed compared to a nonpolar solvent if the PET is at play in the relaxation process.

As can be seen in Fig. 8, 4a indeed demonstrates significant solvatochromism where the growth of the low-energy chargetransfer bands is accompanied by the increase of solvent polarity. On the contrary, 2a had limited response to solvent polarity. As such, the solvatochromism of $\mathbf{4 a}$ in conjunction with the DFT results provides evidence of the presence of PET in phospholelipids with suitable FMO arrangements.
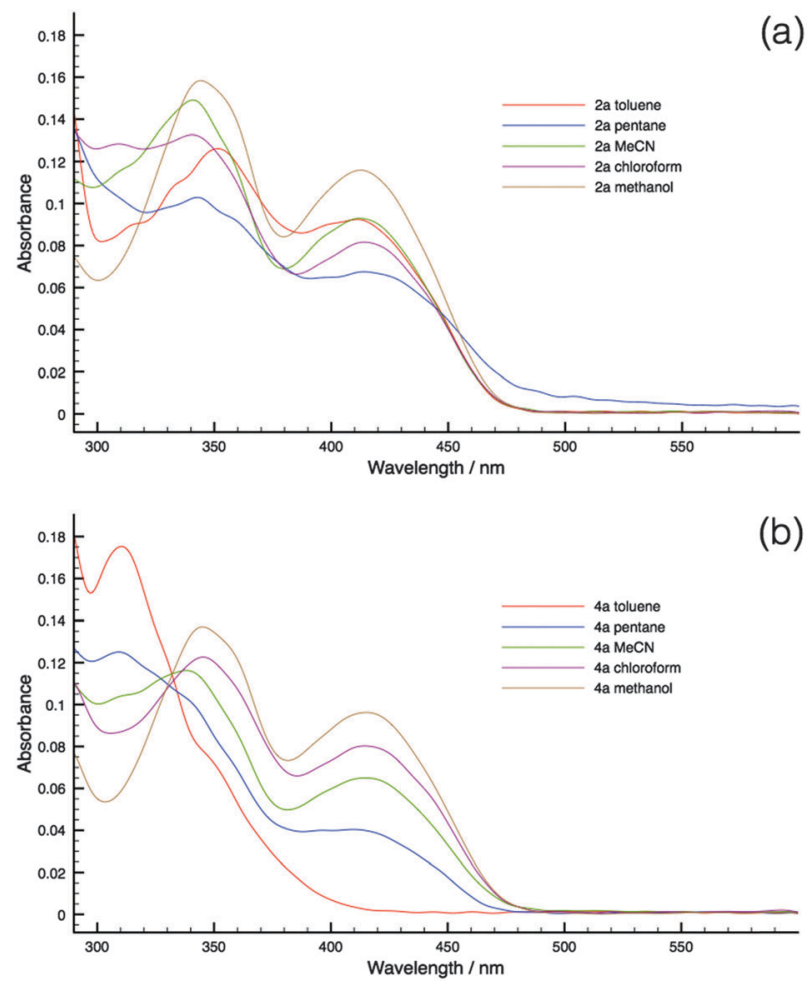

Fig. 8 Solvatochromism of $\mathbf{2 a}$ (top) and $\mathbf{4 a}$ (bottom). All experiments were conducted at $10^{-5} \mathrm{M}$ to mitigate effect of aggregation. (a) Absorption of $\mathbf{2 a}$ in various solvents; (b) absorption of $\mathbf{4 a}$ in various solvents, note the disappearance of the high-energy band at $300 \mathrm{~nm}$ and the appearance of the low energy bands at $350 \mathrm{~nm}$ and $420 \mathrm{~nm}$, respectively. 


\section{Self-organization and AIEE}

As discussed above, the AIEE of phosphole-lipids relies heavily on the intramolecular rotation of the benzyl moiety. Therefore, self-organization of the molecules both in solution and in the solid state plays an important role in tuning the AIEE properties of the compounds. To better understand and further explore and leverage this important relationship, the self-organization of the phosphole-lipids was studied with an array of methods.

Concentration-dependent NMR studies and solution fluorescence. The molecular self-organization of the compounds in solution was studied by concentration-dependent ${ }^{31} \mathrm{P}$ and ${ }^{1} \mathrm{H}$ NMR spectroscopy (see ESI $\dagger$ for spectra).

As can be seen in Table 3 , the ${ }^{31} \mathrm{P}$ NMR signal shows distinct shifts as a function of both concentration and species investigated. As concentration differences lead to conformational changes at the benzylic methylene, ${ }^{4}$ the shift of the ${ }^{31} \mathrm{P}$ NMR signals was correspondingly assigned to conformational variations.

With a shift of only $77 \mathrm{ppb}, \mathbf{4 a}\left(3,5-\mathrm{C}_{12}\right.$ chains) experiences the least changes. This small response to a relatively large concentration range $\left(10^{-3}-10^{-1} \mathrm{M}\right)$ indicates a more rigid conformational environment around the phosphorus center, where the rotation of the benzylic methylene group is restricted. This rigidity of $4 \mathbf{a}$ in solution was also confirmed by the fluorescence quantum yield of the compound in solution. While theory predicted $4 \mathbf{a}$ to have limited fluorescence intensity in solution, in actuality the compound exhibited significant fluorescence in solution $\left(\phi_{\mathrm{PL}}=\right.$ $13 \%$ ). Compound $\mathbf{4 b}$, the octyl analogue of $\mathbf{4 a}$ displays comparable fluorescence $\left(\phi_{\mathrm{PL}}=10 \%\right)$. The rigidity of the molecule along with the free rotation of the alkoxy substituent (vide supra) combined, give the 4-series of compounds increased solution fluorescence as compared to other phosphole-lipids.

By contrast, the ${ }^{31} \mathrm{P}$ NMR signal of $3 \mathbf{a}\left(3,4-\mathrm{C}_{12}\right.$ chains) experiences a shift of $360 \mathrm{ppb}$, indicating a considerably more flexible conformational environment. In this case, this flexibility was mirrored by the non-emissive nature of the compound in solution; the octyl analogue $3 \mathbf{b}$ was also non-emissive in solution.

While $2 \mathbf{a}\left(4-\mathrm{C}_{12}\right.$ chain) was comparable to $3 \mathbf{a}$ in terms of ${ }^{31} \mathrm{P}$ NMR shift (232 ppb), the solution fluorescence of the compound was quite intense $\left(\phi_{\mathrm{PL}}=26 \%\right)$ and that of $2 \mathbf{b}\left(4-\mathrm{C}_{8}\right.$ chain $)$ was equally strong. According to the theoretical prediction, the PET process was suppressed in this compound. As such, the high fluorescent quantum yields of the 2-series confirm the result of the DFT-calculated results.

While ${ }^{31} \mathrm{P}$ NMR spectroscopy revealed the intramolecular interactions of the compounds in solution, ${ }^{1} \mathrm{H}$ NMR spectroscopy was able to provide key information on the intermolecular $\pi-\pi$ interactions of the compounds at various concentrations. The benzo-proton $\mathrm{H}_{\mathrm{a}}$ was selected for examination due to its

Table 3 Concentration-dependent ${ }^{31} \mathrm{P}$ NMR shifts of the phospholelipids

\begin{tabular}{llll}
\hline Compound & $\delta^{31} \mathrm{P}\left(10^{-1} \mathrm{M}\right) / \mathrm{ppm}$ & $\delta^{31} \mathrm{P}\left(10^{-3} \mathrm{M}\right) / \mathrm{ppm}$ & $\Delta \delta^{31} \mathrm{P} / \mathrm{ppb}$ \\
\hline 2a & 19.6769 & 19.9097 & 233 \\
3a & 20.4754 & 20.8383 & 363 \\
4a & 20.5358 & 20.6126 & 77
\end{tabular}

a

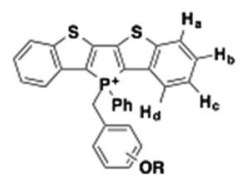

b
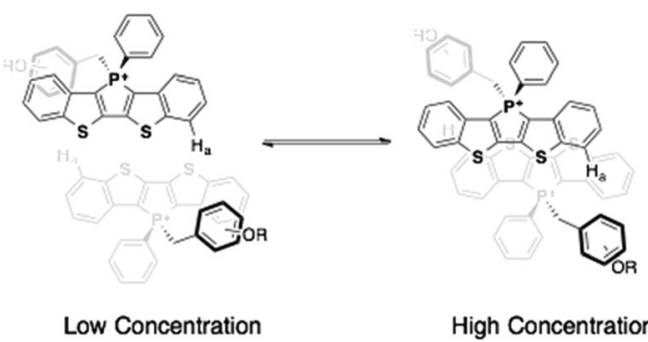

High Concentration

Fig. 9 (a) Proton assignments on the benzo moiety; (b) the proposed dimerization process of phosphole-lipids. Note the position of proton $\mathrm{H}_{\mathrm{a}}$.

potential for response to intermolecular $\pi-\pi$ interactions (Fig. 9). At low concentrations, the molecules appear more monomeric with little intermolecular interactions. With increased concentration, increased intermolecular $\pi$-interactions between two phosphole head groups occur (Fig. 9b). Since in almost all phosphole systems $\pi$ stacking almost always exists in a slip-stack fashion, proton $\mathrm{H}_{\mathrm{a}}$ should experience the most change in its chemical environment with increased $\pi-\pi$ interaction. ${ }^{10}$

The results of the concentration-dependent ${ }^{1} \mathrm{H}$ NMR spectroscopy studies are summarized below. As can be seen in Table 4, 4a displayed the least amount of changes to concentration variation. This is in line with the ${ }^{31} \mathrm{P}$ NMR spectroscopy studies and confirms the rigidity of the compounds in solution. Even at rather high concentration $\left(10^{-1} \mathrm{M}\right)$, 4a still exhibited significant monomeric characteristics. The concentration response of 3a clearly contrasts with $\mathbf{4 a}$. This prominent response of $3 \mathbf{a}$ also confirms the ${ }^{31} \mathrm{P}$ NMR results and indicates a highly flexible system, where the rotation of the benzyl group can easily accommodate the dimerization of the compounds. Compound $2 \mathrm{a}$ also appears to be more flexible than $\mathbf{4 a}$, albeit to a lesser degree than $\mathbf{3 a}$.

The results of concentration-dependent ${ }^{31} \mathrm{P}$ and ${ }^{1} \mathrm{H}$ NMR studies demonstrate empirically that the attachment pattern of the alkyl chains has considerable impact on the solution selfassembly process. The 3,5-bisalkyl arrangement (4a) displays significant rigidity and resistance to intermolecular interactions in solution, while the 3,4-bisalkyl and 4-alkyl arrangement appear to be rather flexible in their scaffold. Despite the fact this difference in rigidity being reflected by the solution emission properties of these compounds, the newly synthesized phosphole-lipids were also studied in the solid state to better understand the mechanism for these self-assembly processes.

Solid-state self-organization and fluorescence. The selforganization of the compounds in the solid state was studied

Table 4 Concentration-dependent ${ }^{1} \mathrm{H}$ NMR shifts of $\mathrm{H}_{\mathrm{a}}$ in the phosphole-lipids

\begin{tabular}{llll}
\hline Compound & $\delta^{1} \mathrm{H}\left(10^{-1} \mathrm{M}\right) / \mathrm{ppm}$ & $\delta^{1} \mathrm{H}\left(10^{-3} \mathrm{M}\right) / \mathrm{ppm}$ & $\Delta \delta^{1} \mathrm{H} / \mathrm{ppb}$ \\
\hline 2a & 8.1214 & 8.1411 & 20 \\
3a & 8.1066 & 8.1819 & 75 \\
4a & 8.2226 & 8.2080 & 15
\end{tabular}


Table 5 Self-assembly features and solid-state fluorescence quantum yields of phosphole-lipids

\begin{tabular}{lll}
\hline Compound & Self-assembly features & Solid-state $\phi_{\mathrm{PL}}{ }^{a}(\%)$ \\
\hline 1b & Viscous liquid/liquid crystal & 0 \\
2a & Amorphous & 5 \\
2b & Soft crystal/liquid crystal & 3 \\
3a & Amorphous & 0 \\
3b & Amorphous & 9 \\
4a & Crystalline & 33 \\
4b & Crystalline & 19 \\
4a-DS & Soft crystal/liquid crystal & 11 \\
${ }^{a}$ Absolute, determined using an integrating sphere.
\end{tabular}

by differential scanning calorimetry (DSC), polarized optical microscopy (POM) and powder X-ray diffractometry (PXRD). The results are summarized in Table 5 (see ESI $\dagger$ for details on thermograms, POM pictures, and powder diffractograms).

As can be seen in Table 5, the self-assembly features of the newly synthesized phosphole-lipids range from the highly crystalline 4-series of compounds to the highly isotropic $1 \mathbf{b}$ species. As a result, the fluorescence of the compounds in the solid state also varies significantly according to the differences in morphology. In general, crystalline compounds display higher fluorescence intensity due to the close packing in the crystalline phase. When crystallinity decreases, so does solid-state fluorescence.

Compound $\mathbf{1 b}$, when newly prepared, appeared to be a highly viscous liquid at room temperature, however, which solidified after several months. While the eventual solid displayed certain degrees of long-range order, as evident by PXRD (see ESI $\dagger$ ), it is its liquidity that dominates the self-assembly of the compound on a more accessible time scale. This highly disordered feature was also found in another short-chain-decorated phosphole species in an earlier study. ${ }^{11}$ With truncated chain length and enlarged conjugated head group, both the shape anisotropy of the molecule and charge density decrease. Since these forces are integral in the ordering of the compounds in aggregation, decreased shape anisotropy and charge density are likely the cause for the high liquidity of $\mathbf{1 b}$. Like its previously studied cousin $\mathbf{1 a}$, the species was essentially non-emissive in the aggregated state $\left(\phi_{\mathrm{PL}}=0.17 \%\right)$.

While 2a was amorphous in nature, $2 \mathbf{b}$ displayed some soft crystal/liquid crystal features as evident by it PXRD results. The low solid-state fluorescence of the compounds is, however, more likely due to ACQ rather than the AIEE effect. This conclusion is based on the particular FMOs arrangements where the PET effect was suppressed (Fig. 6, M2).

While also being amorphous solids, akin to 2a, films of the 3-series can be obtained by drop casting and were studied by scanning electron microscopy (SEM, Fig. 10); films formed by both $3 \mathbf{a}$ and $\mathbf{3 b}$ appear to be of similar thickness $(\sim 20 \mu \mathrm{m}$, see ESI $\dagger$ ). In both cases, amorphous defects were found evenly distributed over the entire surface. However, whereas the film of 3a appeared to be relatively homogeneous, rectangular crystallites can be found embedded through out the surface of the film of $\mathbf{3} \mathbf{b}$. The increased crystallinity of $\mathbf{3} \mathbf{b}$ was reflected by increased solid-state fluorescence quantum yield of $\mathbf{3 b}$ $\left(\phi_{\mathrm{PL}}=9 \%\right)$ as compared to $3 \mathbf{a}\left(\phi_{\mathrm{PL}}=0 \%\right)$. However, due to

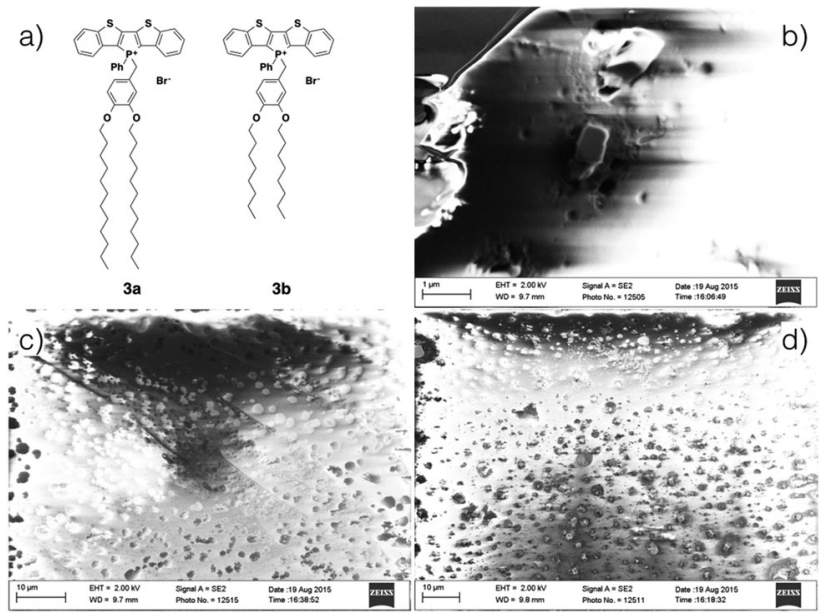

Fig. 10 Scanning electron microscopy of 3-series of phosphole-lipids. (a) Phosphole-lipids $\mathbf{3 a}$ and $\mathbf{3 b}$; (b) crystallites on the surface of $\mathbf{3 b}$ film; (c) amorphous surface of $\mathbf{3 a}$ film; (d) amorphous surface of $\mathbf{3 b}$ film with crystallites on the surface.

the diminutive size of the crystallites, PXRD of the film did not support any increased crystallinity for $\mathbf{3 b}$.

Contrary to the amorphous nature of the 2-and 3-series, the crystallinity of the $\mathbf{4}$-series was confirmed by the relatively sharp melting and crystallization transitions on DSC, large crystalline domains on POM and the prominent sharp peaks in PXRD graphs (see ESI $\dagger$ ). This significantly increased crystallinity of the 4-series is the likely reason for its considerable solid-state fluorescence (Table 5). In fact, $\mathbf{4 a}$ and $\mathbf{4 b}$ proved to be two of the most highly emissive phosphole-lipid species observed to date $\left(\mathbf{4 a} \phi_{\mathrm{PL}}=33 \%, \mathbf{4} \mathbf{b} \phi_{\mathrm{PL}}=19 \%\right)$. Furthermore, we were able to obtain single crystals of $4 \mathbf{a}$ by slow evaporation of solvent mixture (dichloromethane and hexane) at room temperature (Fig. 11).

Due to the thermal disorder generally imparted by the highly flexible dodecyl chains, reports of single crystal X-ray crystallographic data of compounds possessing multiple dodecyl substituents are few and far between. Therefore, the single crystal X-ray data of $4 \mathbf{a}$ afforded us a unique opportunity in understanding the selfassembly feature of the phosphole-lipid system.‡

One of the most significant features of $\mathbf{4 a}$ in the crystalline state is the anti-parallel arrangement of the two dodecyl chains. Contrary to our expectation that the chains would lie parallel to each other and form a linear molecule with polar and non-polar regions on each end, the two dodecyl chains in crystalline $\mathbf{4 a}$ adopt a 'split' stance with the chains pointing away from each other (Fig. 11a). In a recent study, we also reported on the single crystal X-ray data of a 'phosphinine lipid' KP (Fig. 11, KP). ${ }^{12}$

$\ddagger$ Crystal data and structure refinement for $4 \mathrm{a}\left(\mathrm{C}_{53} \mathrm{H}_{70} \mathrm{BrO}_{2} \mathrm{PS}_{2}\right): M_{\mathrm{r}}=914.09$, temperature $=173(2) \mathrm{K}$, triclinic, space group $P \overline{1}, a=9.5341(3), b=14.4282(4), c=$ 18.4781(4) ̊, $\alpha=85.0625(17), \beta=77.5714(16), \gamma=80.3446(13)^{\circ}, V=2443.82(12) \AA^{3}$, $Z=2, \rho_{\text {calc }}=1.242 \mathrm{~g} \mathrm{~cm}^{-3}, \mu=0.998 \mathrm{~mm}^{-1}, F(000)=972.0$, crystal size $=0.070 \times$ $0.050 \times 0.020 \mathrm{~mm}^{3}, \lambda=0.71073 \AA$ А $2 \Theta$ range for data collection $=4.892^{\circ}$ to $55.124^{\circ}$, index ranges $=-12 \leq h \leq 12,-18 \leq k \leq 18,-23 \leq l \leq 24,20775$ measured reflections, $11196\left[R_{\text {int }}=0.0484\right]$ independent reflections, GoF on $F^{2}=1.117, R_{1}=$ $0.0682, \mathrm{w} R_{2}=0.1164[I \geq 2 \sigma(I)], R_{1}=0.1021, \mathrm{w} R_{2}=0.1309$ [for all data], largest difference peak and hole 0.79 and -0.40 e $\AA^{-3}$. 

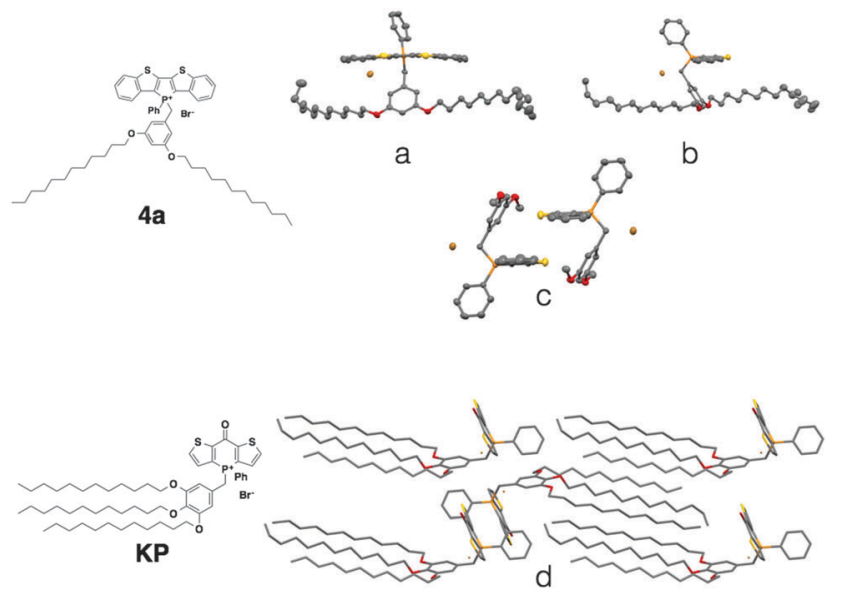

Fig. 11 Single crystal XRD data of phosphole-lipid (4a) and phosphininelipid (KP). (a) and (b) solid-state structures of $\mathbf{4 a}$ as monomers; (c) the intermolecular packing of $\mathbf{4 a}$ (dodecyl chains are truncated for clarity); (d) solid-state packing of $\mathbf{K P}{ }^{9}$

By comparing $\mathbf{4 a}$ and $\mathbf{K P}$ with two different chain attachment patterns (3,5- and 3,4,5-), we can better understand the peculiar geometry of the 3,5-chain arrangement in $\mathbf{4 a}$. While the dodecyl chains in KP were aligned parallel to each other in the solid state (Fig. 11d), the dodecyl chains in $\mathbf{4 a}$ are arranged antiparallel to each other (Fig. 11a). This difference in chain arrangement can be attributed to the lack of van-der-Waals interchain interaction in 4a. The presence of the 4-dodecyl substituents in KP very likely acts as a 'glue' to pull the other two chains together resulting in a parallel arrangement. This particular arrangement of the dodecyl chain in crystalline $\mathbf{4 a}$ can also shed light on the self-assembly processes of the compound in solution (Fig. 11b, Tables 2 and 3). Without an alkyl substituent in the 4-position, the 3- and 5-dodecyl chains are more or less free floating in solution. From the crystallographic data, it can be deduced that the dodecyl chains would be found primarily on the periphery of the molecule, creating a barrier preventing the approach of another molecule (Fig. 12). It is reasonable to believe this is the basis for the apparent reluctance of $\mathbf{4 a}$ to aggregate in solution.

Another important feature of $\mathbf{4 a}$ is the absence of $\pi-\pi$ interactions in $\mathbf{4 a}$ (Fig. 11c), which is commonly found in related phosphole compounds. In most of the other non-lipid phospholes, ${ }^{6,10}$ including closely related ionic model compound $\mathbf{M 1},{ }^{4 c} \pi-\pi$ interactions in the solid state are predominantly of the intermolecular variety where the phosphole $\pi$-scaffolds arrange in a face-to-face fashion. In 4a however, intermolecular $\pi$-stacking interactions between the phosphole scaffolds are almost completely suppressed (Fig. 11c).

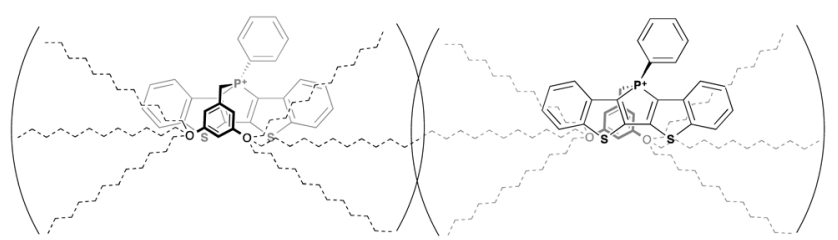

Fig. 12 Proposed mechanism for intermolecular repulsion of 4 a in solution.
Instead of the $\pi-\pi$ dimers commonly observed in other dithienophosphole systems, $\mathbf{4 a}$ appears to remain monomeric, even in the crystalline state. This remarkable 'rigidity' of the molecule was also mirrored in the concentration-dependent ${ }^{31} \mathrm{P}$ NMR studies discussed previously. Although the corresponding $\mathbf{4 b}$ also appears crystalline, crystals of sufficient quality for singlecrystal X-ray crystallography could not be obtained.

Effect of the counter anion. To mediate the crystallinity and introduce amphiphilic features to the self-assembly of $\mathbf{4 a}$, the bromide anion was substituted for the more flexible dodecylsulfate. Whereas $\mathbf{4 a}$ is highly crystalline, the increased thermal disorder introduced by the dodecylsulfate anion results in a decreased crystallinity of 4a-DS in the solid state. The differential thermogram of 4a-DS, shows one extra thermal transition close to the crystal melting transition, suggesting additional phase transitions apart from the melting/crystallization transition (see ESI $\dagger$ ). Moreover, the addition of the dodecylsulfate anion resulted in significantly smaller domains observed via POM when heated (Fig. 13). However, due to the diminutive nature of the domains, visual assignment of phases was unfortunately unsuccessful. PXRD results also confirmed the decreased crystallinity of compound (broad high angle 'bumps' instead of sharp peaks, see ESI $\dagger$ ). The decreased crystallinity of 4a-DS also resulted in the decreased solid-state fluorescence of the

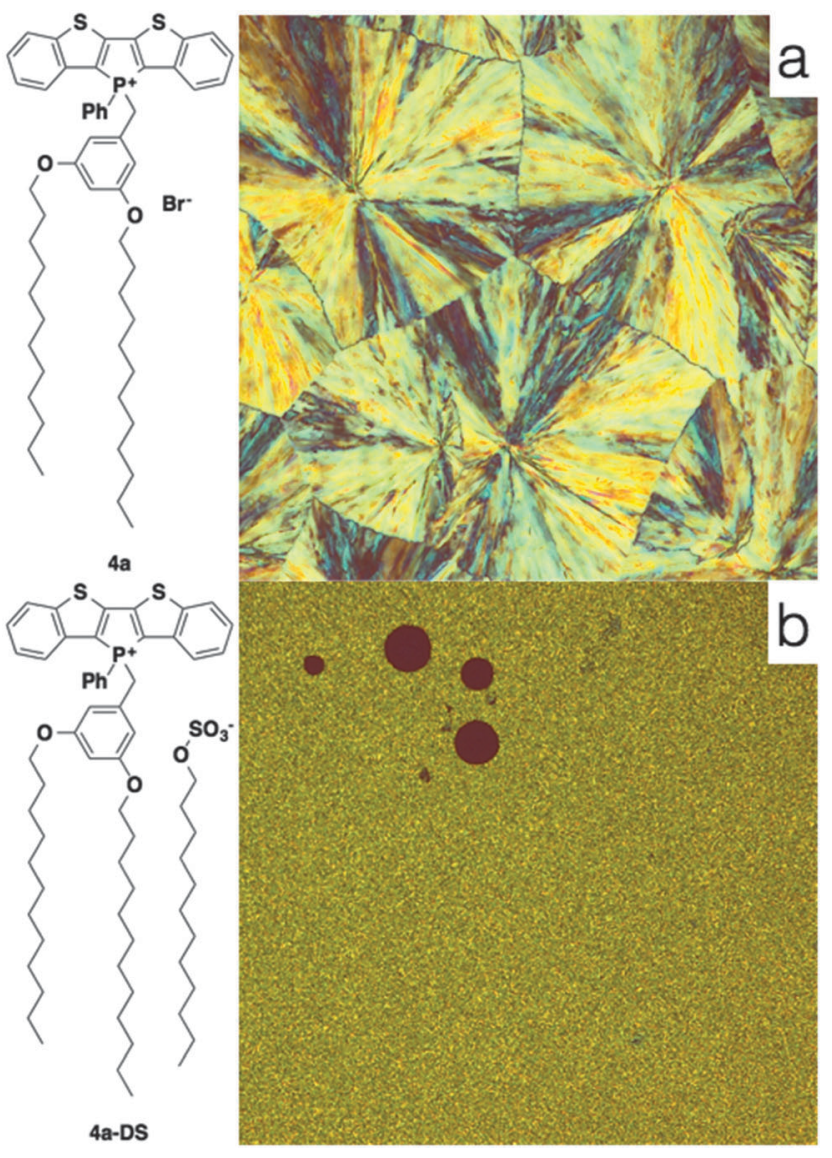

Fig. 13 Polarized optical microscopy of $4 a$ (a) and 4a-DS (b). Note the differences in domain size between the two micrograph. 
compound. Due to the high solution fluorescence quantum yield of 4a-DS $\left(\phi_{\mathrm{PL}}=16 \%\right)$, the addition of the dodecylsulfate anion was enough to partially switch off the solid-state fluorescence and therefore the AIEE of this compound. This is likely due to the increased thermal disorder in the solid state introduced by the flexible dodecylsulfate anion leading to non-radiative relaxation pathways in the solid state. The result of the dodecylsulfate species 4a-DS provided us with another unique avenue in tuning the self-organization and opto-electronic features of the phosphole-lipid system.

\section{Conclusions}

In this study, we successfully demonstrated the tuning of AIEE features of di(benzothieno)phosphole-lipids by self-assembly. Theoretical calculations proved to be an important and reliable tool for predicting and rationalization the opto-electronic properties of the phosphole-lipids. By varying attachment patterns of the alkyl chains on the benzyl moiety, we were able to achieve both crystalline and amorphous self-assembly of the molecules in the solid state. When regarding the theoretical, photophysical and self-assembly results of all the newly synthesized phospholelipids, it can be seen that the self-assembly and related AIEE of phosphole-lipids are based on the delicate balance of all the forces involved in the process, such as ionic interaction, van der Waals interactions, $\pi$ stacking, and sterics. Within the phosphole-lipid system, the benzyl moiety plays an important role in both the self-assembly and the photophysics. In terms of photophysics, by varying the number and electronic nature of the substituent, the orbital energies of the benzyl moiety can be tuned relative independently of the phosphole head groups. By adjusting the donor strength of the benzyl moiety, we can turn on/off the AIEE of the compounds. In terms of the self-assembly, the chain length and attachment patterns determine the self-assembly feature of the compounds. The 4-position on the benzyl moiety, in particular, can be used as a switch for crystalline(4a)/amorphous(3a) features and, moreover, to restrict the potential for free rotation of the neighboring alkoxy groups, which has a considerable effect on the AIEE of the system. While the effect of the chain length is more complicated, it can be said that by varying the chain length we can introduce crystallinity (3a vs. $\mathbf{3 b}$ ) or liquidity (1a $v s$. 1b) and the related photophysical properties. Finally, the dodecylsulfate anion provided us a convenient avenue for tuning the self-assembly feature of our system without drastically altering the intrinsic energy of the chromophores involved. Through this study, we can now begin to assemble the toolbox for rationally designing and building self-assembled phosphole-lipid chromophores with targeted properties.

\section{Acknowledgements}

Financial support by NSERC of Canada and the Canada Foundation for Innovation (CFI) is gratefully acknowledged. We also thank Alberta Innovates - Technology Futures for a graduate scholarship
(Z. W.). Thanks to Profs. T. Sutherland and C. C. Ling for helpful discussion and the use of the polarized optical microscope.

\section{Notes and references}

1 (a) T. Baumgartner and R. Reáu, Chem. Rev., 2006, 106, 4681-4727; (b) T. Baumgartner, Acc. Chem. Res., 2014, 47, 1613-1622; (c) C. Romero-Nieto and T. Baumgartner, Synlett, 2013, 920-937; (d) M. Stolar and T. Baumgartner, Chem. - Asian J., 2014, 9, 1212-1225; (e) F. Jäkle, Chem. Rev., 2010, 110, 3985-4022; $(f)$ A. Wakamiya and S. Yamaguchi, Bull. Chem. Soc. Jpn., 2015, 88, 1357-1377; ( $g$ ) S. Yamaguchi and K. Tamao, in The Chemistry of Organic Silicon Compounds, ed. Z. Rappoport and T. Appeloig, John Wiley \& Sons, Chichester, 2001, vol. 3, pp. 641-694.

2 (a) T. Baumgartner, T. Neumann and B. Wirges, Angew. Chem., Int. Ed., 2004, 43, 6197-6201; (b) Y. Dienes, M. Eggenstein, T. Neumann, U. Englert and T. Baumgartner, Dalton Trans., 2006, 1424-1433; (c) S. Durben, Y. Dienes and T. Baumgartner, Org. Lett., 2006, 8, 5893-5896; (d) Y. Ren and T. Baumgartner, J. Am. Chem. Soc., 2011, 133, 1328-1340.

3 (a) Y. Ren and T. Baumgartner, Dalton Trans., 2012, 41, 7792-7800; (b) Z. Wang and T. Baumgartner, Chem. Rec., 2015, 15, 199-217.

4 (a) Y. Ren, W. H. Kan, M. A. Henderson, P. G. Bomben, C. P. Berlinguette, V. Thangadurai and T. Baumgartner, J. Am. Chem. Soc., 2011, 133, 17014-17026; (b) Y. Ren, W. H. Kan, V. Thangadurai and T. Baumgartner, Angew. Chem., Int. Ed., 2012, 51, 3964-3968; (c) Y. Ren and T. Baumgartner, Inorg. Chem., 2012, 51, 2669-2678.

5 (a) Y. Hong, J. W. Y. Lam and B. Z. Tang, Chem. Commun., 2009, 4332-4353; (b) Y. Hong, J. W. Y. Lam and B. Z. Tang, Chem. Soc. Rev., 2011, 40, 5361-5388; (c) Z. Zhao, B. He and B. Z. Tang, Chem. Sci., 2015, 6, 5347-5365.

6 Y. Dienes, M. Eggenstein, T. Kárpáti, T. C. Sutherland, L. Nyulászi and T. Baumgartner, Chem. - Eur. J., 2008, 14, 9878-9889.

7 L.-C. Lee and Y. Zhao, J. Am. Chem. Soc., 2014, 136, 5579-5582. 8 (a) A. C. Nieuwkerk, A. T. M. Marcelis, A. Koudijs and E. J. R. Sudhölter, Liebigs Ann., 1997, 8, 1719-1724; (b) M. R. Bozorgmehr, M. Saberi and H. Chegini, J. Mol. Liq., 2014, 199, 184-189.

9 M. J. Frisch, et al., Gaussian09, Revision C.01, Gaussian, Inc., Wallingford, CT, 2009, (see ESI $\dagger$ for full reference).

10 (a) T. Baumgartner, W. Bergmans, T. Kárpáti, T. Neumann, M. Nieger and L. Nyulászi, Chem. - Eur. J., 2005, 11, 4687-4699; (b) Y. Ren, Y. Dienes, S. Hettel, M. Parvez, B. Hoge and T. Baumgartner, Organometallics, 2009, 28, 734-740; (c) S. Durben and T. Baumgartner, Angew. Chem., Int. Ed., 2011, 50, 7948-7952; (d) X. He, J.-B. Lin, W. H. Kan, P. Dong, S. Trudel and T. Baumgartner, Adv. Funct. Mater., 2014, 24, 897-906.

11 Y. Ren, F. Biegger and T. Baumgartner, J. Phys. Chem. C, 2013, 117, 4748-4758.

12 X. He, J.-B. Lin, W. H. Kan and T. Baumgartner, Angew. Chem., Int. Ed., 2013, 52, 8990-8994. 